\title{
EL POLEN DE EUCALYPTUS Y SU INCIDENCIA EN LA ATMÓSFERA DE VIGO (N.O. ESPAÑA)
}

\author{
F. Javier RODRÍGUEZ-RAJO, Mª Victoria JATO y Mª Carmen SEIJO
}

\begin{abstract}
RESUMEN. El polen de Eucalyptus y su incidencia en la atmósfera de Vigo (N.O. España). Eucalyptus globulus Labill. es la especie arbórea de la familia Myrtaceae, más frecuente en los alrededores de la ciudad de Vigo formando abundantes y densas masas forestales. Aunque su polen se considera moderadamente alergénico, en áreas en las que, como consecuencia de repoblación forestal ocupa extensiones importantes, puede llegar a causar problemas alérgicos.

Entre los años 1995 y 2000 se ha realizado el estudio aerobiológico en la ciudad de Vigo mediante un captador LANZONI VPPS 2000 situado en la margen derecha de la ría de Vigo (42 $14^{\circ} 15^{\prime \prime} \mathrm{N}, 8^{\circ} 43^{\prime} 30^{\prime \prime} \mathrm{W}$ ). La cantidad de granos de polen anual de Myrtaceae varía ampliamente, alternándose años de concentraciones bajas con otros de valores elevados que representan entre un 1 y un $3 \%$ del polen total anual. El valor más elevado se detectó en 1997 con 1.147 granos de polen. Se encuentra en la atmósfera de Vigo durante todo el año, aunque las concentraciones más importantes se producen generalmente durante los meses de marzo y abril. Asimismo, a lo largo del día los máximos de concentración tienen lugar entre las 15 y las 17 horas. Finalmente se ha realizado un análisis estadístico para poner de manifiesto la posible correlación existente entre las concentraciones de polen y los principales parámetros meteorológicos. El viento procedente del $\mathrm{N}$-NE fue el parámetro con el que se obtuvo correlación positiva y significativa de forma constante, mientras que esta fue negativa con la humedad relativa.
\end{abstract}

Palabras clave. Meteorología, Myrtaceae, Polen, Eucalyptus, Vigo.

ABSTRACT. The Eucalyptus pollen and its incidence in the atmosphere of Vigo (N.W. Spain). Eucalyptus globulus Labill. is the most frequent arboreal species of the Myrtaceae family in the surroundings of the city of Vigo, and it constitues abundant and dense forestal masses. Although its pollen can be considered as a moderated allergen, in areas in which Eucalyptus was used in extended reafforestations, it can cause allergenic problems.

From 1995 to 2000 a Hirst-type volumetric sampler (Hirst, 1952), LANZONI VPPS 2000 placed in the left bank of the Vigo estuary $\left(42^{\circ} 14^{\prime} 15^{\prime \prime} \mathrm{N}, 8^{\circ} 43^{\prime} 30^{\prime \prime} \mathrm{W}\right)$ was used for collecting airborne pollen. From year to year important oscillations in the annual total pollen was observed, alternating years of high concentrations with other of lower values. Eucalyptus pollen counts represent a percentage that vary between $1 \%$ to $3 \%$ of the total pollen, the high quantity being collected in 1997 with 1,147 pollen grains. The Myrtaceae pollen appears in the atmosphere of Vigo all over the year, although the more important concentrations are registered during March and April. Moreover, maximum daily concentrations occur between 17.00 and 18.00 hours. Finally, a statistical analysis has been carried out in order to search for the possible correlations between pollen concentration and the main meteorological parameters. The N-NE wind and relative humidity were the parameters that show the highest correlation coefficients, positive and negative respectively.

Key words. Meteorology, Myrtaceae, Pollen, Eucalyptus, Vigo.

Este trabajo ha sido realizado gracias a los proyectos MAB 97-0457.C07-03 D.G.I.C.Y.T., Xunta de Galicia PGIDT00MAM38301 y la financiación de la Consellería de Medio Ambiente de la Xunta de Galicia. 


\section{INTRODUCCIÓN}

La familia Myrtaceae se encuentra representada en Vigo por dos géneros que forman parte de la flora ornamental de la ciudad: Myrtus y Eucalyptus. El primero, representado por la especie Myrtus communis L., es utilizado como arbusto para la formación de setos y el segundo como árbol en parques y jardines, correspondiendo a este último la mayor parte de los granos de polen identificados como pertenecientes a esta familia. De las 2 especies de Eucalyptus presentes en el área, Eucalyptus globulus Labill. es la más frecuente en los alrededores de la zona de estudio formando abundantes y densas masas forestales, mientras que Eucalyptus camaldulensis Dehen. se encuentra de forma ocasional. El área de distribución natural de Eucalyptus se corresponde con la región biogeográfica Austral, encontrándose en Australia la mayor diversidad de especies. Desde sus lugares de origen ha sido distribuido por todo el mundo, especialmente en zonas de clima mediterráneo, subtropical y tropical, en donde encuentra las condiciones adecuadas para su desarrollo.

En España su área preferente de cultivo es la cornisa cantábrica, Galicia y el sudoeste peninsular. Quercus robur L., es el árbol que de forma natural debiera ser dominante en los bosques caducifolios que representan las cabezas de serie de la vegetación potencial del sector Galaico-Portugués. Sin embargo, los montes de la provincia de Pontevedra se encuentran dedicados mayoritariamente a la producción maderera, basada prácticamente en el cultivo de dos especies, Pinus pinaster Aiton y Eucalyptus globulus Labill., de crecimiento rápido y las primeras en germinar después de los incendios. Actualmente en España hay en total alrededor de $550.000 \mathrm{Ha}$ de eucalipto, de las que el $27 \%$ pertenecen a Galicia. El 20\% de sus ejemplares más longevos se encuentran en la provincia de Pontevedra (Bermúdez \& Touza, 2000).
El polen de los géneros Myrtus y Eucalyptus se considera como causante de reacciones alérgicas. (Lewis et al. (1983), Domínguez et al. (1984) y Subiza et al. (1986, 1988, 1995) califican a Eucalyptus camaldulensis como moderadamente alergógeno. Asimismo, se ha apuntado la posibilidad de que presente reactividad cruzada con otros tipos polínicos (Domínguez et al., 1984).

En general las concentraciones alcanzadas por este tipo polínico son bajas por lo que su correlación con problemas alérgicos es difícil de evaluar. No obstante, en las zonas en las que Eucalyptus ocupa amplias extensiones, puede llegar a causar problemas alérgicos. En este sentido, Belmonte et al. (1998) en la ciudad de Vigo, encuentran que un $3 \%$ de los pacientes sensibles a algún tipo de polen lo son al de este género, constituyendo el octavo inductor principal de polinosis. Asimismo en Ourense, ciudad situada a $100 \mathrm{Km}$ de Vigo, el porcentaje de sensibilización en pruebas cutáneas con extractos de polen de Eucalyptus es inferior al 8\% (Arenas et al., 1996).

Según la bibliografía consultada, no se ha detectado este tipo polínico en la atmósfera de la parte Norte y Centro de España, y únicamente en Cataluña y localidades costeras de Andalucía se ha citado su presencia en cantidades importantes (Belmonte et al., 1999; González et al., 1999; Trigo et al., 1998). La abundancia de este árbol en la zona litoral gallega, la posible incidencia de su polen como inductor de procesos alérgicos y la escasez de estudios aerobiológicos del polen de Myrtaceae, son las razones por las cuales consideramos que sería importante establecer las épocas del año en las que se alcanzan las concentraciones más elevadas y las horas del día en las que se registran los valores máximos. Asimismo se realiza un análisis estadístico que permite conocer las correlaciones existentes entre las concentraciones polínicas y las principales variables meteorológicas. 


\section{MATERIAL Y MÉTODOS}

La ciudad de Vigo se encuentra situada en el noroeste de España, enclavada en la ría del mismo nombre (fig. 1). De las especies presentes en el área de estudio mencionadas en el apartado anterior, la más abundante es Eucalyptus globulus Labill., género al que pertenecen la mayor parte de los granos de polen contabilizados.

Se ha utilizado un captador volumétrico de impacto activo tipo Hirst (Hirst, 1952) modelo LANZONI VPPS 2000 ubicado en la margen izquierda de la ría de Vigo $\left(42^{\circ} 14^{\prime} 15^{\prime \prime} \mathrm{N}, 8^{\circ} 43^{\prime} 30^{\prime \prime} \mathrm{W}\right)$ a una altura de 50 $\mathrm{m}$ sobre el nivel del mar. El período de estudio incluye desde el 1 de enero de 1995 hasta el 31 de diciembre del 2000.

Como superficie de captura se ha utilizado una cinta Melinex impregnada en silicona al $2 \%$ en tetracloruro de carbono. Los valores medios diarios se han expresado como número de granos de polen por metro cúbico de aire. Para la identificación y recuento de los granos de polen se ha utilizado un microscopio NIKON OPTIPHOT II provisto de una lente 40X/0.95, siguiendo la metodología propuesta por la R.E.A., basado en la realización de cuatro barridos longitudinales a lo largo de la preparación (Domínguez et al., 1992). Para la identificación de los granos de polen se han tenido en cuenta guías de referencia (Valdés et al., 1987, y Spieksma et al., 1993), así como la palinoteca del grupo de aerobiología de la Facultad de Ciencias de Ourense.

La temperatura media anual en Vigo es $14,9^{\circ} \mathrm{C}$, con una media de las temperaturas máximas de $18,8^{\circ} \mathrm{C}$ y de las mínimas de $11^{\circ} \mathrm{C}$. La precipitación media anual es de $1.412 \mathrm{~mm}$, siguiendo un patrón irregular a lo largo del año, ya que los meses de julio y agosto son normalmente bastante secos, con una precipitación total menor de $40 \mathrm{~mm}$ (Carballeira et al., 1983).

Para la obtención del período de
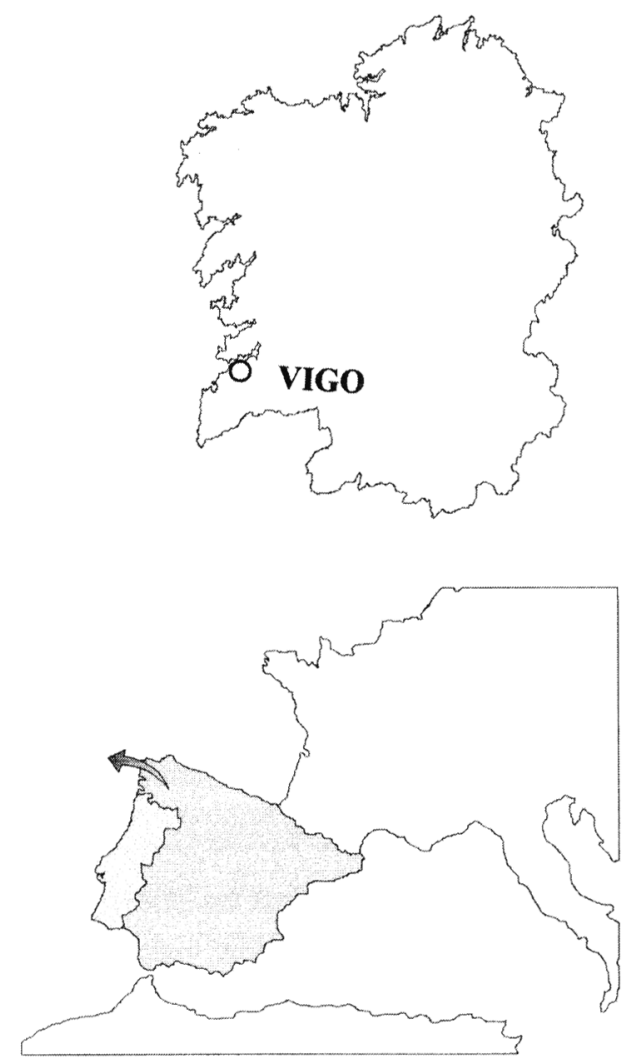

Figura 1. Localización de la zona de estudio. Location of the zone of study.

polinización principal (PPP), se ha tenido en cuenta el método descrito por Andersen (1991), quién establece que dicho período abarca desde el momento en que la suma de las concentraciones medias diarias de polen alcanza el $2,5 \%$ del total anual, hasta que su suma llegue al $97,5 \%$, es decir, la estación polínica comprenderá el período de tiempo en que se recoge el $95 \%$ del total anual de este tipo polínico.

Se ha tratado de encontrar un modelo que refleje la fluctuación de la concentración polínica a lo largo del día. Para ello, una vez establecido el período de polinización descrito 
anteriormente, se ha calculado la media diaria de concentración polínica durante el mismo y se han seleccionado aquellos días en los que se registran valores superiores al promedio calculado, eliminando los días en los que se han registrado precipitaciones debido a su efecto de lavado atmosférico. Con los días resultantes, cuyo número total osciló entre 24 y 62 según el año estudiado, se ha calculado la media de concentración cada dos horas, expresando los datos posteriormente en $\%$ frente a la concentración total de ese "día ideal" (Galan et al., 1991).

Finalmente, se ha utilizado el test de correlación de Spearman con el objetivo de comprobar el grado de asociación existente entre las concentraciones de polen de Eucalyptus y los principales factores meteorológicos: lluvia (mm.), humedad relativa (\%), horas de sol (horas), temperatura máxima, mínima y media $\left({ }^{\circ} \mathrm{C}\right)$ y la dirección del viento (\%). Estos datos meteorológicos fueron suministrados por el Instituto Nacional de Meteorología.

La correlación se ha establecido teniendo en cuenta el total del PPP, el período prepico (el cual abarca desde el inicio de la estación hasta el día en el que se registra la concentración media diaria máxima) y el postpico (periodo que comprende desde el momento en el que se registra la concentración media diaria máxima hasta el final del PPP).

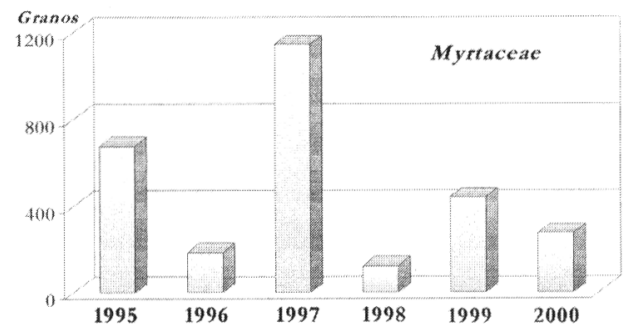

Figura 2. Polen total anual durante los años de muestreo $\left(\mathrm{m}^{3}\right)$. Total annual pollen during the years of the study $\left(\mathrm{m}^{3}\right)$.

\section{RESULTADOS}

Los valores de polen total anual registrado a lo largo de los años que comprende el estudio varían ampliamente (fig. 2), alternándose años de concentraciones elevadas (1995, 1997 y 1999) con otros de valores más bajos (1996, 1998 y 2000). El año en el que se recogió una mayor cantidad de polen de Myrtaceae fue 1997 con 1.147 granos de polen. El porcentaje que este tipo polínico representa frente al polen total anual osciló entre un 1 y un $3 \%$.

Las concentraciones medias registradas en los años estudiados se representan en la figura 3. Aunque con valores bajos, el polen de Eucalyptus estuvo presente en la atmósfera de Vigo durante la mayor parte del año, si bien lo hace de manera constante en el invierno y la primavera (fig. 3). Las concentraciones más elevadas se producen generalmente durante los meses de marzo y abril. La gráfica de la figura 4 representa la evolución de las concentraciones obtenidas como media para los años 1995-2000.

Las principales características del Período de Polinización Principal utilizando el método del 95\% (Andersen, 1991), tales como la fecha de inicio y final, el número de días que incluye, la media de granos de polen, el valor máximo, el día en el que este tiene lugar, la cantidad de polen registrado a lo largo del mismo y el porcentaje que este tipo polínico representa frente al total, se muestran en la tabla 1.

En la figura 5 se representa el modelo de comportamiento horario, representando en ella los valores medios para cada dos horas obtenidos para cada uno de los años de muestreo y el hallado como medio para el conjunto de los cinco años. Este modelo refleja concentraciones máximas durante la tarde, en concreto entre las 15 y 21 horas. Posteriormente estas decaen a lo largo de las últimas horas del día y se mantienen de forma constante en niveles bajos durante la madrugada, con un ligero incremento entre las 3-4 horas, 

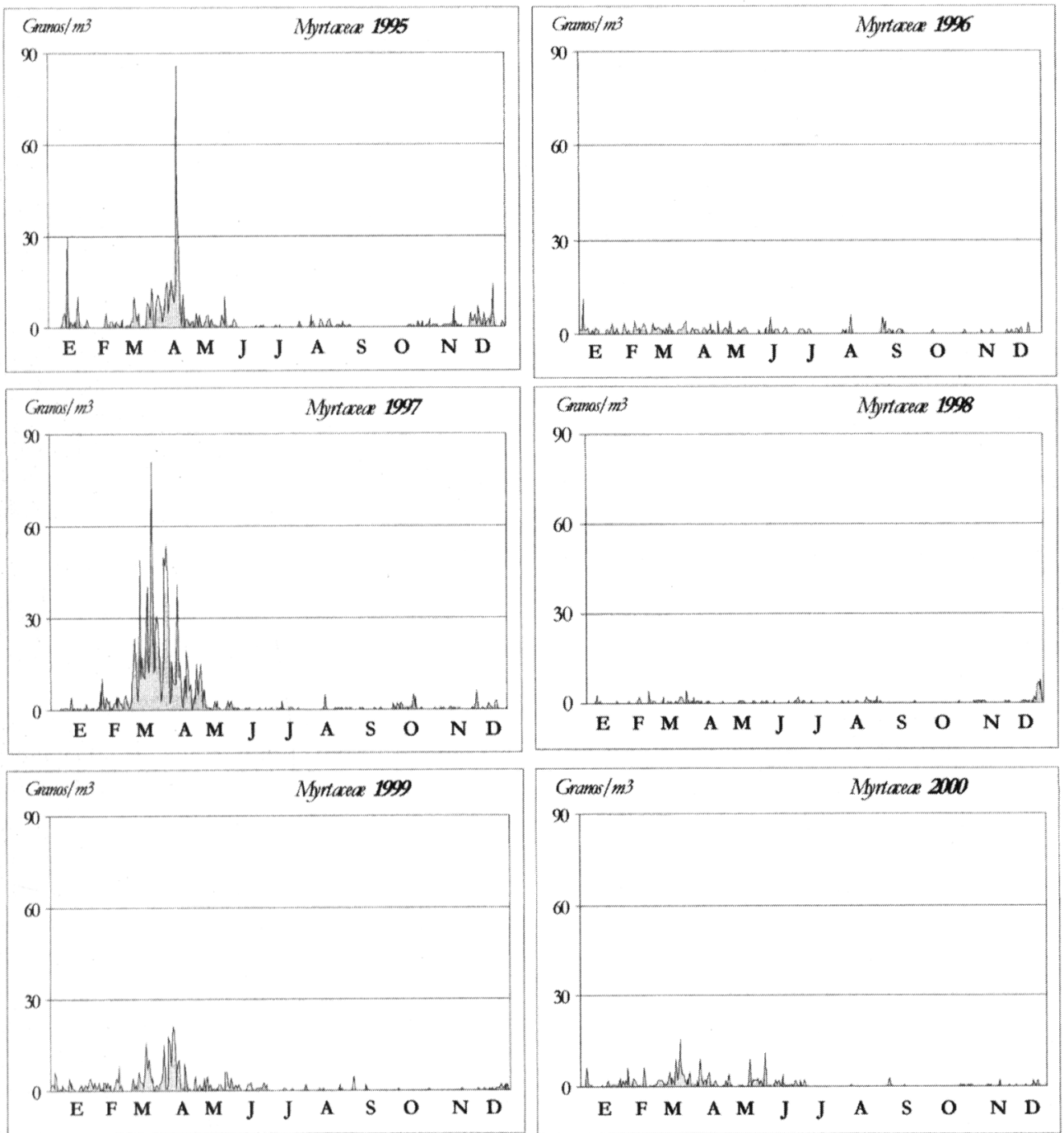

Figura 3. Variación estacional de las concentraciones medias diarias a lo largo de los años de estudio. Daily seasonal variation during the years of the study. 


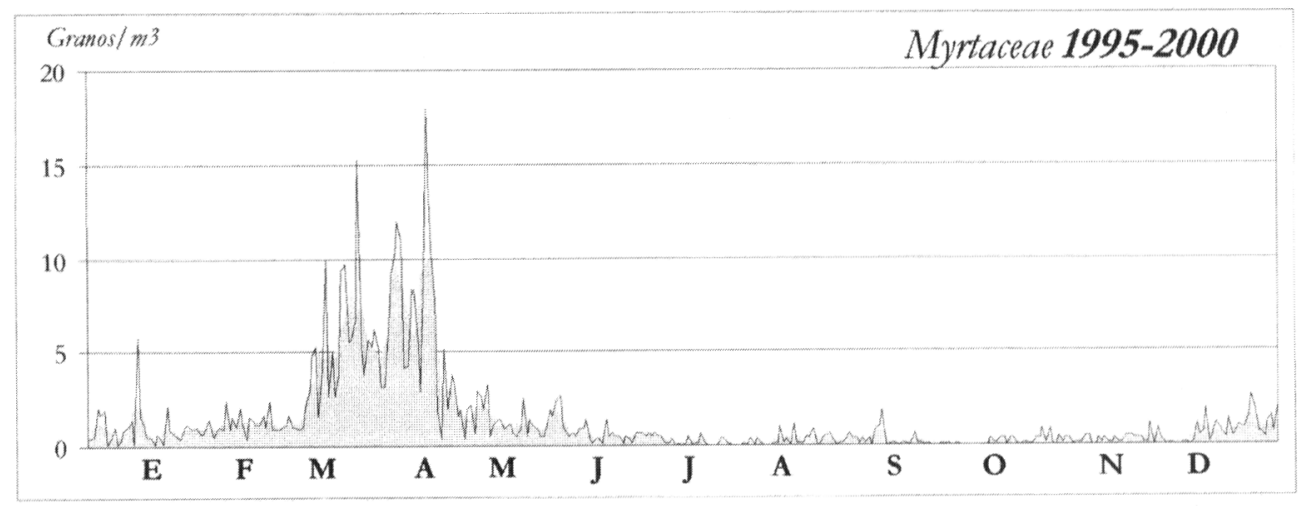

Figura 4. Modelo de variación estacional medio. Mean daily seasonal variation.

aumentando de forma rápida a partir del mediodía.

Finalmente en la tabla 2 se representan los coeficientes de correlación de Spearman obtenidos entre las concentraciones de polen de Eucalyptus y los principales parámetros meteorológicos en cada uno de los años y para el conjunto de los mismos. En primer lugar se reflejan los calculados teniendo en cuenta la estación polínica, y a continuación los obtenidos para el periodo prepico y el postpico. Se ha elegido este estadístico no paramétrico debido a que los datos de polen no se distribuyen de una manera normal, tal como se expresa en la tabla 3, en la cual se tienen en consideración tanto el test de normalidad de KolmogorovSmirnov como el de Lilliefors.

Teniendo en cuenta el conjunto de los años de estudio, las correlaciones resultaron significativas (nivel de significación del 99\%) con la mayor parte de los parámetros meteorológicos. La correlación fue negativa para la humedad relativa, lluvia, temperatura mínima, viento en calma y viento del S-SW, y positiva con el viento del N-NE y el recorrido del mismo.

La ausencia de valores del coeficiente de correlación en los periodos prepico de 1998 y postpico de 1998 y 2000, se debe al escaso número de datos con los que se ha realizado el análisis debido a las concentraciones tan bajas de polen de Eucalyptus registradas durante dichos periodos.

\section{DISCUSIÓN}

La presencia del polen de Eucalyptus en la ciudad de Vigo es prácticamente constante a lo largo del año, siendo el taxon que permanece en la atmósfera de la ciudad un número más elevado de días (Rodríguez-Rajo, 2000). El hecho de no presentar una estacionalidad marcada como los árboles de la zona templada, podría ser consecuencia de ser un árbol de reciente introducción, ya que las primeras semillas fueron enviadas por el padre Rosendo Salvado (De la Lama, 1978) a su familia de Tuy en el año 1846. Por ello, a pesar de que la comunidad gallega y en especial las zonas costeras, poseen unas condiciones climatológicas adecuadas para el desarrollo del eucalipto, este aún se encuentra en plena fase de adaptación. Consecuentemente su polinización sigue el habitual comportamiento de los árboles del hemisferio norte y además es capaz de liberar polen a la atmósfera en otras épocas del año como recordatorio de su 


\begin{tabular}{lcccccc}
\hline Myrtaceae & 1995 & 1996 & 1997 & 1998 & 1999 & 2000 \\
\hline Fecha de Inicio & 16-enero & 4-enero & 12-febrero & 9-enero & 5-enero & 6-enero \\
Fecha del Final & 21-diciembre & 12-diciembre & 20-octubre & 28-diciembre & 30-agosto & 21-diciembre \\
Duración (días) & 340 & 344 & 251 & 354 & 238 & 351 \\
Media en el P.P.P. $\left(\mathrm{gr}^{\prime} / \mathrm{m}^{3}\right)$ & 2 & 1 & 4 & 1 & 2 & 1 \\
Máximo $\left(\mathrm{gr} . \mathrm{m}^{3}\right)$ & 86 & 11 & 81 & 7 & 21 & 16 \\
Día del máximo & 14 -abril & 4 -enero & 24 -marzo & 27-diciembre & 9-abril & 18 -marzo \\
Polen Total en el P.P.P. & 661 & 175 & 1094 & 113 & 414 & 262 \\
Polen Total en la estación & 679 & 185 & 1147 & 123 & 441 & 276 \\
\hline \% sobre el total anual & $\mathbf{2}$ & $\mathbf{1}$ & $\mathbf{3}$ & $<\mathbf{1}$ & $\mathbf{2}$ & $\mathbf{1}$
\end{tabular}

Tabla 1. Características del PPP de Myrtaceace (95\%). Characteristics of the Myrtaceae Main Pollination Period (95\%).

procedencia del hemisferio sur. Este patrón lo presentan los árboles más jóvenes, ya que los primeros ejemplares introducidos, los cuales son a su vez los mayores productores de polen, florecen en su mayor parte únicamente durante la primavera. En este sentido es una constante a lo largo del período de estudio que las concentraciones más elevadas se suelan producir de forma clara durante los meses de marzo y abril (fig. 3).

El comportamiento de floración bianual descrito para árboles de floración invernalprimaveral tales como Alnus, Betula y Quercus (Andersen, 1980; Nilsson \& Persson, 1981; Emberlin et al., 1993; Jäger et al., 1991; ElGhazaly et al.,1993, Spieksma et al., 1995, Latorre, 1999; Rodríguez-Rajo, 2000) en el que se alternan años en los que el árbol prima un afán reproductor frente a otros en los que se centra en el crecimiento vegetativo, también es seguido en el caso de Myrtaceae. Así, durante el período de estudio se alternan estaciones en las que se recogen hasta 1.147 granos de polen con años en los que no se superó el valor de 276 (fig. 3).

El comportamiento bianual que este tipo polínico describe, también se ve influenciado por las condiciones meteorológicas reinantes durante el período de polinización (Dahl \& Stradhede, 1996), ya que la intensa floración producida durante 1997 coincide con escasas precipitaciones en los meses de marzo y abril, y por el contrario las concentraciones registradas para los años 1996, 1998 y 2000 coincidieron con períodos de bajas temperaturas e intensas lluvias acaecidas durante el período de floración. Durante el año 2000 han sido especialmente importantes las precipitaciones registradas durante el mes de abril y la primera quincena de mayo, ya que se alcanzaron un total de 477 y $191 \mathrm{~mm}$ respectivamente (hasta 10 veces superiores a las registradas en otros años). En este periodo de tiempo únicamente se encontraron 4 días con ausencia de precipitaciones. Por otra parte la correlación especialmente negativa con la humedad relativa, dificulta también su transporte haciendo disminuir las concentraciones polínicas.

En cuanto a las concentraciones máximas a lo largo del período de estudio, los años en los que es más abundante, las medias diarias alcanzan hasta 80 granos $/ \mathrm{m}^{3}$ y por el contrario los años de bajos registros no se superan los 11 granos $/ \mathrm{m}^{3}$. El valor medio diario máximo se alcanzó el 14 de abril de 1995, día en el que se registraron 86 granos $/ \mathrm{m}^{3}$. La media de granos de polen registrada en el PPP es siempre muy baja y no supera los 4 granos $/ \mathrm{m}^{3}$ (tab. 1).

Como corresponde a la amplia distribución que Eucalyptus globulus posee en la zona de estudio, los valores alcanzados en la ciudad de Vigo duplican a los apuntados para otras localidades gallegas (Aira et al., 1999), alcanzándose en España niveles similares 

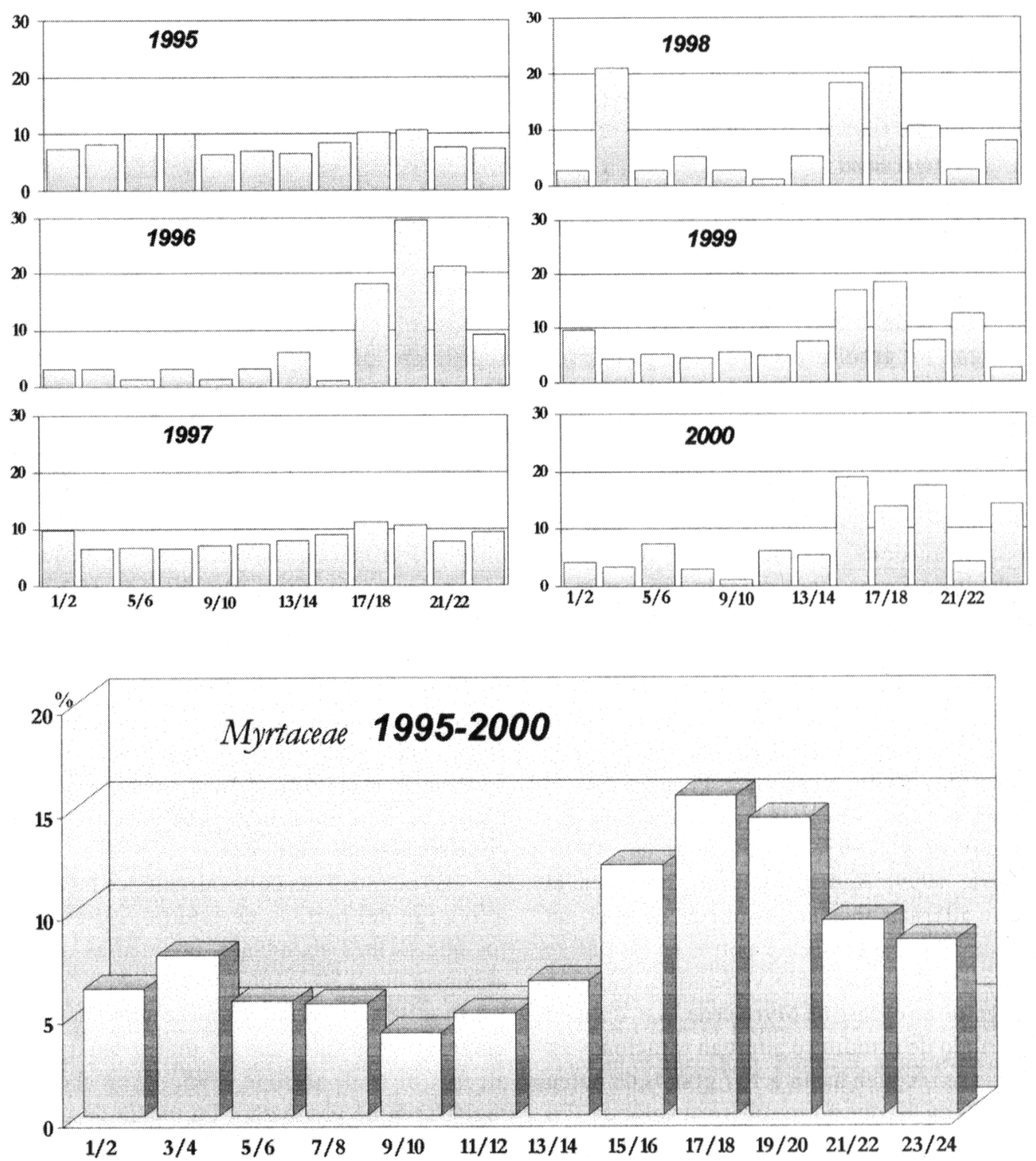

Figura 5. Modelo de variación intradiurno a lo largo de los años de muestreo (\%). Intradiurnal variation during the years of the study (\%). 

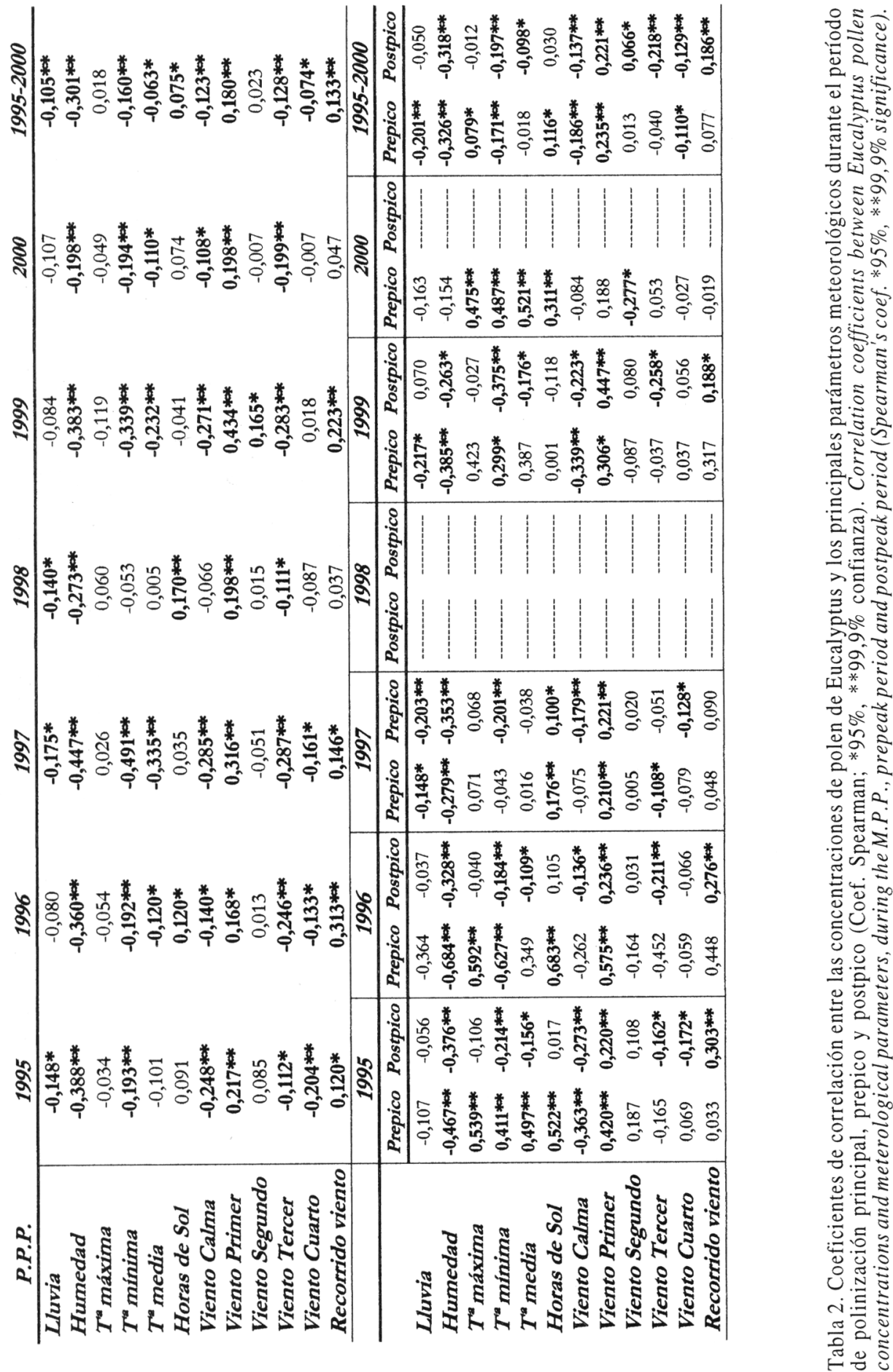


\begin{tabular}{llcl}
\hline Myrtaceae & \multicolumn{2}{l}{ Kolmogorov-Smirnov } & Lilliefors \\
\hline & $\mathbf{d}$ & $\mathbf{p}$ & $\mathbf{p}$ \\
$\mathbf{1 9 9 5 - 2 0 0 0}$ & 0.328 & $<0.01$ & $<0.01$ \\
$\mathbf{1 9 9 5}$ & 0.316 & $<0.01$ & $<0.01$ \\
$\mathbf{1 9 9 6}$ & 0.420 & $<0.01$ & $<0.01$ \\
$\mathbf{1 9 9 7}$ & 0.251 & $<0.01$ & $<0.01$ \\
$\mathbf{1 9 9 8}$ & 0.463 & $<0.01$ & $<0.01$ \\
$\mathbf{1 9 9 9}$ & 0.261 & $<0.01$ & $<0.01$ \\
$\mathbf{2 0 0 0}$ & 0.389 & $<0.01$ & $<0.01$ \\
\hline
\end{tabular}

Tabla 3.Valores de los test de normalidad de Kolmogorov-Smirnov y Lilliefors. Kolmogorov-Smirnov and Lilliefors normality test values.

únicamente en las ciudades de Málaga (Trigo et al., 1998) y Huelva (González et al., 1999), en las que se registraron alrededor de 1.100 granos de polen total durante 1997.

El patrón de variación horaria de concentraciones de polen de eucalipto, si tenemos en cuenta el modelo medio obtenido, refleja que las concentraciones máximas se producen durante la mitad de la tarde, y en concreto entre las 17-18 horas momento en el que se registra un $16 \%$ del total diario (fig. 5). Un comportamiento similar sigue el polen de esta familia cuando consideramos cada año por separado aunque con ligeras modificaciones, ya que durante el año 2000 el pico se adelanta dos horas y durante 1996 se retrasa dos horas, momento en el que se recoge el $30 \%$ del total de polen diario. Además podemos observar cómo los picos son más acusados en los dos años que presentan una menor concentración total anual de polen (1996 y 1998). En localidades del sur de España como Córdoba (Galán et al., 1991) o Málaga (Recio, 1995) se ha obtenido un patrón irregular, con varios picos a lo largo del día.

Teniendo en cuenta el análisis de correlación, éstas resultaron significativas (nivel de significación del 99\%) de forma positiva únicamente con el viento procedente del N-NE y con el recorrido del mismo. Por otra parte se encontró una correlación negativa para la lluvia, la humedad, temperatura mínima y cuando el viento se encuentra en calma o procede del mar (tab. 2). En general, este patrón de comportamiento se mantiene estable cuando consideramos los años de estudio por separado así como al realizar el estudio con los valores prepico y postpico. Es importante señalar que debido a las bajas concentraciones que este tipo polínico presenta y a que se encuentra en floración durante la mayor parte del año, es muy dificil el establecer un PPP para el polen de esta familia, sobre todo en los años con valores bajos. En este sentido, los valores de los coeficientes durante los años en los que las concentraciones son más elevadas (1995, 1997 y 1999), son superiores. En 1999 y 1995 la correlación con la temperatura mínima, según se considere el PPP o el prepico es de signo contrario, siendo negativa en el primer caso y positiva en el segundo.

Asimismo, cuando el viento procede de la dirección NE (primer cuadrante), donde existen grandes áreas de plantaciones de Eucalyptus con fines madereros, aumentan los valores de este polen y por el contrario disminuyen cuando el viento procede del SW-N (el mar). La existencia de correlación negativa con el viento en calma y positiva con el recorrido del viento podría indicar que este polen es liberado en zonas cercanas a la ciudad y transportado hacia la misma.

En trabajos llevados a cabo en Málaga se observa que el parámetro con correlaciones 
más elevadas es la temperatura, seguido de las horas de sol (Recio, 1995), aunque en este caso la localización geográfica en la que se encuentra dicha ciudad, con unas temperaturas más elevadas y un aporte pluviométrico menor al registrado en Vigo, podría ser un factor importante que explicase las diferencias de comportamiento de este tipo polínico.

\section{CONCLUSIONES}

Myrtaceae es una de las familias mejor representadas en la flora de los alrededores de la ciudad de Vigo, ya que la especie Eucalyptus globulus Labill. es muy frecuente y forma abundantes y densas masas forestales. A pesar de ser un taxon de polinización eminentemente entomófila, aparece representado en la atmósfera de la zona de estudio en cantidades considerables. El total de granos de polen presentes en la atmósfera de la ciudad a lo largo de un año, presentó variaciones considerables, oscilando entre años con elevadas concentraciones frente a otros que no sobrepasaron los 275 granos de polen. El porcentaje que representa frente al polen total contabilizado a lo largo del año no supera un $3 \%$.

Presenta un período de polinización muy largo, iniciándose durante el mes de diciembre y manteniéndose en la atmósfera la práctica totalidad del año (hasta 351 días en el año 2000). De todas formas su comportamiento a lo largo del año no es homogéneo, existiendo periodos en los que su presencia en la atmósfera es más acusada. En este sentido hasta un $64 \%$ del total anual se concentra durante los meses de marzo y abril.

Asimismo, a lo largo del día se observa que los máximos de concentración tienen lugar entre las 17 y 18 horas. Finalmente podemos señalar que la concentración atmosférica de Myrtaceae presenta una correlación positiva con el viento procedente del N-NE y el recorrido del mismo, y una correlación negativa para la lluvia, la humedad, temperatura media, mínima y cuando el viento procede del mar. Todas ellas presentan un nivel de significación del $99 \%$.

AGRADECIMIENTOS. Los autores agradecen al Servicio de Medio Ambiente del Concello de Vigo su importante colaboración.

\section{BIBLIOGRAFÍA}

AIRA M.J., A. DOPAZO y A. ARGIBAY -1999Aeropalinología en Galicia: Estación de Santiago de Compostela (1998). Rea 5: 119112.

ANDERSEN, T. -1991- A model to predict the beginning of the pollen season. Grana 30: 269275.

ANDERSEN, S. Th. -1980- Influence of climatic variation on pollen season severity in windpollinated trees and herbs. Grana 19: 47-52.

ARENAS, L., C. GONZÁLEZ, J.M. TABARÉS, I. IGLESIAS, M.V. JATO y J. MÉNDEZ -1996Sensibilización cutánea a pólenes en pacientes afectos de rinoconjuntivitis-asma en la población de Ourense en el año 1994-1995. Proceedings of the $I^{\text {st }}$ European Symposio on Aerobiology, CA'96. Santiago.

BELMONTE, J., J.M. ROURE \& X. MARCH 1998- Aerobiology of Vigo, North-Western Spain: Atmospheric pollen spectrum and annual dynamics of the most important taxa, and their clinical importance for allergy. Aerobiologia 14: 155-162.

BELMONTE, J., M. VENDRELL y J.M. ROURE 1999- Aerobiología en Cataluña: estación de Barcelona (1999). Rea 5: 79-82.

BERMÚDEZ, J. y M. TOUZA -2000- Las cifras del tercer inventario forestal en Galicia y su incidencia en la industria de la transformación de la madera. Cis-Madera 2000, 4: 6-24.

CARBALLEIRA, A., C. DEVESA, R. RETUERTO, E. SANTILLÁN y E. UCIEDA -1983Bioclimatología de Galicia. Fund. Barrié de la Maza, La Coruña: 391.

Dahl, A. \& Strandhede, S. -1996- Predicting the 
intensity of the birch pollen season. Aerobiologia, 12: 97-106.

DE LA LAMA, G. -1978- Atlas del eucalipto. Instituto Nacional de investigaciones Agrarias, Ministerio de Agricultura (TOMO V). Madrid. DOMÍNGUEZ, E., J.L. UBERA y C. GALÁN 1984- Polen alergógeno de Córdoba. Publicaciones del Monte de Piedad y Caja de Ahorros de Ronda. Córdoba.

DOMÍNGUEZ, E., C. GALÁN, F.E. VILLAMANDOS \& F. INFANTE -1992Handling and evaluation of the data from the aerobiological sampling. Monografías Rea-Ean 1: $1-18$.

EL-GHAZALY, G., P. EL-GHAZALY, K. LARSSON \& S. NILSON -1993- Comparison of airborne pollen grains in Huddinge and Stockholm, Sweden. Aerobiología 9: 53-67.

EMBERLIN, J., M. SAVAGE \& R. WOODMAN 1993- Annual variations in the concentrations of Betula pollen in the London area, 1961-1990. Grana, 29: 301-309.

GALÁN, C., R. TORMO, J. CUEVAS, F. INFANTE \& E. DOMÍNGUEZ - 1991- Theoretical daily variation patterns of airborne pollen in the South-west of Spain. Grana 30: 201-209.

GÓNZALEZ, F.J., J. MORALES, P. CANDAU, C. TOMÁS y A.M. PÉREZ TELLO -1999Aerobiología en Andalucía: estación de Sevilla (1998). Rea 4: 49-52.

HIRST, J.M. -1952- An automatic volumetric sporetrap. Ann. Appl. Biol. 36: 257-265.

JÄGER, S., F.TH. SPIEKSMA \& N. NOLARD 1991- Fluctuations and trends in airborne concentrations of some abundant pollen types, monitored at Vienna, Leiden and Brussels. Grana 30: 309-312.

LATORRE, F. -1999- Differences between airborne pollen and flowering phenology of urban trees with reference to production, dispersal and interannual climate variability. Aerobiologia 15: 131-141.

LEWIS, W.H., P. VINAY \& V.E. ZENGER - 1983Airborne and allergenic pollen of North America. The Johns Hopkins Univers. Press. 254. Baltimore.

NILSSON, S. \& S. PERSSON -1981- Tree pollen spectra in the Stockholm region (Sweden), 19731980. Grana 20: 179-182.

RECIO, M. -1995-Análisis polínico de la atmósfera de Málaga (1991-1994). Relación con los parámetros meteorológicos. Tesis Doctoral. Universidad de Málaga.

RODRÍGUEZ-RAJO, F.J. -2000- El polen como fuente de contaminación ambiental en la ciudad de Vigo. Tesis Doctoral. Universidad de Vigo. SPIEKSMA, F. TH., N. NOLARD, G. FRENGUELLI \& D. VAN MOERBEKE - 1993. Polen atmosférico en Europa. UCB, Bruselas.

SPIEKSMA, F. TH., J.C. EMBERLIN, M HJELMROOS, S. JÄGER \& R.M. LEUSCHNER -1995- Atmospheric birch (Betula) pollen in Europe: Trends and fluctuations in annual quantities and the starting dates of the seasons. Grana 34: 51-57.

SUBIZA, J., JEREZ, M., JIMÉNEZ, J.A., NARGANES, J.M., CABRERA, M., VARELA, S. \& SUBIZA, E. -1995- Allergenic pollen pollinosis in Madrid. J. Allergy Clin. Immunol. 96: $15-23$

TRIGO, M.M., M. RECIO, F.J. TORO Y B CABEZUDO - 1998- Aerobiología de Andalucía estación de Málaga. (1995-1998). Rea 3: 33-36

VALDÉS, B., M.J. DÍEZ e I. FERNÁNDEZ, (eds.) -1987-Atlas polínico de Andalucía Occidental. Inst. de desarrollo Regional de la Universidad de Sevilla. Excma. Diputación de Cádiz. Sevilla.

Aceptado para su publicación en julio de 2001

Dirección de los autores. Dpto.. de Biología Vegetal y Ciencias del Suelo. Facultad de Ciencias de Ourense. Campus As Lagoas. E-32004 Ourense (España). 